\title{
Incidência das lesões confundidoras da lesão por pressão em unidade de terapia intensiva oncológica
}

\section{Incidence of injuries confused with pressure injuries in oncology intensive care unit Incidencia de las lesiones confundidoras de la lesión por presiónen unidad de terapia intensiva oncológica}

José Ferreira Pires Júnior ${ }^{1,2^{*}}$, Eline Lima Borges', Josimare Aparecida Otoni Spira²

ORCID IDS

Pires Júnior JF (DD https://orcid.org/0000-0002-6019-0198

Borges EL (iD https://orcid.org/0000-0002-0623-5308

Spira JAO (D) https://orcid.org/0000-0002-6577-0394

\section{COMO CITAR}

Pires Júnior JF, Borges EL, Spira JAO. Incidência das lesões confundidoras da lesão por pressão em unidade de terapia intensiva oncológica. ESTIMA, Braz. J. Enterostomal Ther., 17: e0219. https:// doi.org/10.30886/estima.v17.667 PT

\begin{abstract}
RESUMO
Objetivos: Estimar a incidência das lesões confundidoras (LCS) da lesão por pressão (LP) em unidade de terapia intensiva (UTI) de um hospital oncológico, caracterizar os pacientes e as LCS. Métodos: Estudo de coorte prospectivo realizado em UTI de hospital oncológico de Belo Horizonte, estado de Minas Gerais. A amostra foi composta por 67 pacientes que atenderam aos critérios de inclusão. Os dados foram coletados durante o período de um mês por único pesquisador estomaterapeuta e registrado em formulário elaborado para esse fim. O estudo foi aprovado pelo comitê de ética em pesquisa. Resultados: 58,1\% eram mulheres, 65,1\% idosos, 51,2\% brancos, com predominância de câncer gastrointestinal, 71,6\% em uso de quimioterapia adjuvante, com índice de massa corporal (IMC) abaixo da normalidade, 100\% apresentavam incontinência urinária ou fecal e em uso de fraldas. A incidência das LCS foi 35,6\%, com predomínio da dermatite associada à incontinência (DAI). O tempo médio para o surgimento foi de 11 dias. Conclusão: A incidência das LCs foi elevada e infere-se que possa ter relação com a presença do câncer e o tratamento neoadjuvante, o que requer estudos específicos para elucidar o fato.
\end{abstract}

DESCRITORES: Unidades de terapia intensiva; Oncologia; Ferimentos e lesões; Incidência; Lesão por pressão; Estomaterapia.

1.Universidade Federal de Minas Gerais - Escola de Enfermagem - Departamento de Enfermagem Básica - Belo Horizonte/MG - Brasil.

2.Instituto Mário Penna - Hospital Luxemburgo - Belo Horizonte/MG - Brasil.

Autor correspondente: piresjuniorr@hotmail.com

Recebido: Nov. 21, 2018 | Aceito: Fev. 25, 2019 


\begin{abstract}
Objectives: Estimate the incidence of the confounding lesions $(\mathrm{CL})$ with pressure injuries in an intensive care unit of an oncology hospital, to characterize the patients and the confused injuries. Methods: Prospective cohort study conducted in an adult intensive care unit of an oncology hospital in Belo Horizonte, Minas Gerais. The sample consisted of 67 patients who met the inclusion criteria. The data were collected during the period of one month by single researcher stomatherapist and registered in the form prepared for this purpose. The study was approved by the Research Ethics Committee. Results: $58.1 \%$ were women, $65.1 \%$ were elderly, $51.2 \%$ were white, with gastrointestinal cancer predominating, $71.6 \%$ were using adjuvant chemotherapy, with below-normal body mass index, $100 \%$ had urinary incontinence or fecal and diaper use. The incidence of these injuries was 35.8\%, and the average time for their onset was 11 days after admission. Most patients had skin changes such as maceration, peeling, erythema, dehydration, and edema. Conclusion: We can conclude that the incidence of confused injuries is high in oncology patients admitted to intensive care units. It was possible to describe the demographic and clinical profile of the patients of the sample and to characterize the injuries and the skin around them.
\end{abstract}

DESCRIPTORS: Intensive care units; Medical Oncology; Wounds and injuries; Incidence; Pressure ulcer; Stomatherapy.

\title{
RESUMEN
}

Objetivos: estimar la incidencia de las lesiones confundidoras (LC) de la lesión por presión en unidad de terapia intensiva (UTI) de un hospital oncológico, caracterizar a los pacientes y las LC. Métodos: estudio de cohorte prospectivo realizado en UTI de hospital oncológico de Belo Horizonte, Minas Gerais. La muestra fue compuesta por 67 pacientes que atendieron los criterios de inclusión. Los datos fueron recolectados durante el período de un mes por único investigador estomaterapeuta y registrado en formulario elaborado para este fin. El estudio fue aprobado por el Comité de Ética en Investigación. Resultados: 58,1\% eran mujeres, 65,1\% ancianos, $51,2 \%$ blancos, con predominio del cáncer gastrointestinal, 71,6\% en uso de quimioterapia adyuvante, con índice de masa corporal por debajo de la normalidad, 100\% presentaban incontinencia urinaria o fecal y en uso de pañales. La incidencia de las LC fue 35,6\% con predominio de la dermatitis asociada a incontinencia. El tiempo medio para la aparición fue de 11 días. Conclusión: la incidencia de las LC fue elevada y se infiere que pueda tener relación con la presencia del cáncer y el tratamiento neoadyuvante, lo requiere estudios específicos para elucidar este hecho.

DESCRIPTORES: Unidades de cuidados intensivos; Oncología médica; Heridas y lesiones; Incidencia; Úlcera por presión; Estomaterapia.

\section{INTRODUÇÃO}

Desde o início do século XXI, as lesões de pele, denominadas lesões cutâneas ou simplesmente feridas, têm recebido atenção especial por parte dos profissionais de saúde em decorrência da alta prevalência e incidência. Além disso, causam impacto socioeconômico para os pacientes, familiares, serviços de saúde e sociedade em geral e são responsáveis por afetar negativamente a saúde e a qualidade de vida das pessoas acometidas ${ }^{1}$.

No grupo das lesões que acometem os pacientes internados, destaca-se a lesão por pressão (LP), denominada úlcera por pressão até abril de 2016, quando ocorreu a mudança da nomenclatura pelo órgão americano National Pressure Ulcer Advisory Panel (NPUAP) 2,3. Nessa mesma data, o conceito foi revisado e a LP passou a ser considerada dano localizado na pele e/ou tecido mole subjacente, geralmente sobre proeminência óssea, ou pode ainda estar relacionada a equipamentos médicos ou outro tipo de dispositivo. A lesão pode apresentar-se como pele intacta ou como úlcera aberta e pode ser dolorosa. Ocorre como resultado da exposição à pressão ou da pressão associada ao cisalhamento. A tolerância do tecido mole para a pressão e cisalhamento também pode ser afetada pelo microclima, nutrição, perfusão, doenças associadas e condição do tecido ${ }^{2,3}$.

A multiplicidade de fatores de risco relacionados ao desenvolvimento de LP é, concomitantemente, de outros tipos de lesões cutâneas. Essa situação, muitas vezes, leva os profissionais a confundirem a presença da LP com lesões cutâneas de outras causas. A LP estágio 2 é definida como perda da pele em sua espessura parcial com exposição da derme. Por apresentar-se úmida, com coloração rosa ou vermelha ou como uma bolha intacta ou rompida, esse estágio é confundido com a dermatite associada à incontinência (DAI), à lesão por fricção (LF) e à lesão relacionada a adesivos médicos ${ }^{3}$. No presente estudo, essas lesões serão denominadas lesões confundidoras (LCs) para a ocorrência da LP. Alguns profissionais tendem a classificar as lesões cutâneas como LP. Esse fato resulta na falta de registros institucionais das LCs. 
No Brasil, a partir da publicação da Portaria No 529 de 1 de abril de 2013 pelo Ministério da Saúde 4 a LP passa a ser considerada evento adverso, isso é, um incidente que resulta em dano para o paciente; além disso, sua ocorrência é de notificação compulsória mensal.

Desde 2009, as diretrizes publicadas pela NPUAP e pela European Pressure Ulcer Advisory Panel (EPUAP) reconhecem a DAI como condição distinta à $\mathrm{LP}$ e recomendam que os profissionais assistenciais sejam capacitados para a realização do diagnóstico diferencial ${ }^{2}$.

A DAI é um tipo de dermatite associada à umidade definida como a exposição prolongada da pele ao efluente (urina, fezes, exsudato e suor) com presença de eritema e edema da superfície, podendo ser acompanhada de erosão, flictenas com exsudato seroso ou infecção secundária. Acomete principalmente as regiões perineal, perianal e inguinal ${ }^{5}$.

Outro tipo de LC é a LF, considerada lesão traumática que ocorre com maior frequência nas extremidades superiores e inferiores de pessoas idosas, resultante da fricção ou da combinação de fricção e cisalhamento. Esse dano provoca separação da epiderme da derme e resulta em ferida de espessura parcial ou ocasiona a separação total da epiderme e derme das estruturas subjacentes, sucedendo uma ferida de espessura total ${ }^{6}$.

A terceira lesão do grupo das LCs é a lesão relacionada aos adesivos médicos, cuja definição é a ocorrência em que a hiperemia e/ou outra manifestação de alteração na pele, como vesícula, flictena, erosão e ruptura, persistam por mais de 30 minutos após a remoção do adesivo médico. Em geral, essas lesões estão relacionadas ao trauma mecânico e químico oriundo do uso de desses adesivos médicos ${ }^{7}$.

Embora seja um problema antigo, infere-se que a ocorrência das LCs tem se elevado com o aumento da longevidade da população brasileira e da incidência de doenças crônico-degenerativas, especialmente o câncer. Esse contexto suscita a realização de estudos específicos relacionados a essas lesões, comuns na prática clínica, mas pouco estudadas. Os resultados podem contribuir para a sistematização da nomenclatura das LCs e fomentar a discussão a respeito da prevenção e tratamento.

$\mathrm{Na}$ prática clínica, em várias instituições de saúde do Brasil, ainda persistem dúvidas sobre as causas para o desenvolvimento das LCs e os fatores que atrasam seu processo de cicatrização. Mediante a complexidade apresentada pelos pacientes oncológicos, principalmente aqueles internados em unidade de cuidados intensivos, emerge a questão: qual é a incidência das LCs e as quais são as características dos pacientes que as desenvolvem?

Estudos sobre a ocorrência das lesões por fricção, DAI e as relacionadas a adesivos médicos são escassos, inclusive os epidemiológicos, referentes à incidência e à prevalência dessas lesões, tornando-se inexistentes nas bases de dados quando são relacionadas com os pacientes críticos com câncer.

Essa constatação fomenta a necessidade do desenvolvimento de pesquisas tendo como amostra os pacientes críticos de um hospital oncológico que, devido à própria doença e como consequência dos tratamentos demandados, infere-se que têm risco aumentado para o surgimento dessas lesões. Portanto, os objetivos deste estudo foram estimar a incidência das LCs da LP em uma unidade de terapia intensiva (UTI) de um hospital oncológico e caracterizar os pacientes e as LCs.

\section{MÉTODOS}

Trata-se de estudo piloto de coorte prospectivo. Para a realização da pesquisa, consideraram-se todos os pacientes admitidos na UTI em um hospital oncológico filantrópico de grande porte de Belo Horizonte, estado de Minas Gerais.

A população constituiu-se de 144 pacientes em internação na UTI no mês da pesquisa e a amostra foi do tipo intencional composta por 67 pacientes que atenderam aos critérios de inclusão: idade igual ou superior a 18 anos, permanecer internado na UTI por período mínimo de 48 horas após a primeira avaliação, permitindo, assim, o seguimento com duas avaliações consecutivas da pele durante o período de coleta dos dados. Também foi considerado critério de inclusão apresentar fator de risco para ao menos uma LC; incontinência urinária, fecal ou dupla para DAI; edema para LF; adesivo fixado a qualquer parte do corpo, independentemente de sua intenção, para lesão relacionada a adesivo médico.

Os dados foram coletados por único pesquisador estomaterapeuta durante o período de um mês. Para preenchimento do instrumento de coleta de dados, utilizaram-se informações extraídas do prontuário eletrônico e por meio da inspeção diária da pele dos pacientes no momento da higienização no leito, com intuito de evitar maiores desconfortos aos pacientes.

Para atender aos objetivos propostos, elegeram-se as seguintes variáveis do estudo: LC (DAI, LF, lesão por adesivo) avaliada quanto ao tipo, número, localização e 
características da pele ao redor; variáveis demográficas (idade, sexo e raça/etnia); clínicas [doença que levou à internação na UTI; doenças associadas, hábitos nocivos à saúde (etilismo e tabagismo); medicações em uso na UTI; tratamento neoadjuvante; IMC; hemoglobina; glicemia], presença de edema, uso de adesivo (fita adesiva microporosa ou outro tipo de adesivo), tipo de incontinência (urinária, fecal, mista), dispositivos para controle da incontinência (cateter urinário externo, cateter vesical intermitente, cateter vesical de demora, fralda descartável, dispositivo coletor para estomia intestinal e urinário), cuidados com a pele (pomada à base de óxido de zinco, ácidos graxos essenciais, bepantol, nistatina, cetoconazol), higienização da pele (água + sabão neutro).

Utilizaram-se média, mediana e desvio padrão (DP) para descrição das variáveis numéricas. Já as variáveis categóricas foram sumarizadas por frequências absolutas e relativas e intervalo de confiança de 95\% (95\%CI), quando pertinente.

Para calcular a incidência, considerou-se o número absoluto de casos de pessoas que desenvolveram LC no período da pesquisa dividido pelo número de pacientes com fator de risco (fator de risco para ao menos uma LC: incontinência urinária, fecal ou dupla para DAI; edema para LF; adesivo fixado a qualquer parte do corpo, independentemente de sua intenção, para lesão relacionada a adesivo médico) para LC decorrente de DAI, LF e lesão relacionada a adesivo médico internadas no mesmo período multiplicado por 100 .

O projeto de pesquisa obteve aprovação do comitê de ética da instituição campo do estudo sob o parecer $n^{\circ}$ 2.072.922. A pesquisa respeitou os termos da Resolução no 466 de 12 de dezembro de 2012 do Conselho Nacional de Saúde. O Termo de Consentimento Livre e Esclarecido
(TCLE) foi assinado pelo paciente ou pelo seu responsável legal para participação no estudo.

\section{RESULTADOS}

O estudo consistiu do acompanhamento de 67 pacientes. Desses, 24 desenvolveram pelo menos um tipo de LC, correspondendo à incidência geral de 35,6\%. Considerando cada tipo de LC, encontrou-se incidência de 20,8\% para DAI, 5,9\% para LF e 8,9\% para lesão por adesivo médico. A descrição das variáveis demográficas dos participantes encontra-se na Tabela 1.

Todos os pacientes da amostra tinham câncer, sendo os mais frequentes o gastrointestinal (20/29,9\%), de próstata (12/17,9\%), de mama $(9 / 13,4 \%)$ e do colo do útero (8/11,9\%). Para controle da doença, 71,6\% fizeram uso exclusivamente de quimioterapia, $10,5 \%$ de radioterapia e $17,9 \%$ foram submetidos à quimioterapia e à radioterapia.

A principal causa de internação na UTI foi a insuficiência respiratória aguda (25\%). As doenças associadas mais frequentes apresentadas pelos participantes foram hipertensão arterial sistêmica (32,8\%), diabetes melito (28,3\%), cardiopatia $(14,9 \%)$ e depressão $(13,4 \%)$.

Com relação ao uso de medicamentos, 11,9\% não faziam uso. Os demais participantes utilizavam um ou mais medicamentos, como anti-hipertensivo ou diurético $(33,8 \%)$, sedativos $(32,8 \%)$, analgésicos $(28,8 \%)$, dentre outros.

A maioria dos participantes apresentava índice de massa corporal (IMC) abaixo da normalidade (38/56,7\%) com média de $16,8 \mathrm{~kg} / \mathrm{m}^{2}( \pm 2,1)$, taxa de hemoglobina sérica menor que $12,5 \mathrm{~g} / \mathrm{dL}(49 / 73,1 \%)$ com média de $9,3( \pm 1,5)$ e glicemia capilar maior que $126 \mathrm{mg} / \mathrm{dL}$ (35/52,2\%) com média de $167 \mathrm{md} / \mathrm{dL}( \pm 37)$.

Tabela 1. Características demográficas dos participantes ( $n=67)$, Belo Horizonte, estado de Minas Gerais, 2018.

\begin{tabular}{|c|c|c|c|c|c|}
\hline \multirow{2}{*}{ Variável } & \multirow{2}{*}{ Categoria } & \multicolumn{2}{|c|}{ Lesões confundidoras n (\%) } & \multirow{2}{*}{$\begin{array}{c}\text { Média } \\
\text { (Desvio padrão) }\end{array}$} & \multirow{2}{*}{ Mediana } \\
\hline & & Não $(n=43)$ & $\operatorname{Sim}(n=24)$ & & \\
\hline \multirow{2}{*}{ Sexo } & Feminino & $25(58,1)$ & $15(62,5)$ & \multirow{2}{*}{-} & \multirow{2}{*}{-} \\
\hline & Masculino & $18(41,9)$ & $9(37,5)$ & & \\
\hline \multirow{4}{*}{ Idade (anos) } & $18-30$ & $1(2,3)$ & $0(0,0)$ & \multirow{4}{*}{$68,3(12,4)$} & \multirow{4}{*}{71,0} \\
\hline & $31-59$ & $14(32,6)$ & $5(20,8)$ & & \\
\hline & $60-79$ & $24(55,8)$ & $15(62,5)$ & & \\
\hline & Mais de 80 & $4(9,3)$ & $4(16,7)$ & & \\
\hline \multirow{3}{*}{ Raça/etnia } & Branco & $22(51,2)$ & $12(50,0)$ & \multirow{3}{*}{ - } & \multirow{3}{*}{-} \\
\hline & Preto & $7(16,3)$ & $5(20,8)$ & & \\
\hline & Pardo & $14(32,5)$ & $7(29,2)$ & & \\
\hline
\end{tabular}


A Tabela 2 mostra a distribuição por tipo de lesão dos 24 pacientes que apresentaram LC.

O tempo de surgimento da LC variou de um a 30 dias após internação na UTI, com média de $11,0( \pm 13,9)$ dias e mediana de 5,0 dias. A maioria das lesões $(66,7 \%)$ surgiu com tempo inferior a 10 dias, principalmente as por adesivo médico e fricção.

A maioria das LC era por DAI (14/58,3\%), com localização em região de glúteo (9/64,3\%), perianal (4/28,6\%) e perigenital $(1 / 27,1 \%)$. As seis lesões por adesivo médico estavam nos membros superiores e as quatro lesões por fricção estavam na região da panturrilha (2), escápula (1) e hemitórax (1).

Tabela 2. Tipo de lesão confundidora da amostra $(n=24)$, Belo Horizonte, estado de Minas Gerais, 2018.

\begin{tabular}{|c|c|c|}
\hline Variável & Categoria & n (\%) \\
\hline \multirow{2}{*}{ Lesão por adesivo médico } & $\operatorname{Sim}$ & $6(25,0)$ \\
\hline & Não & $18(75,0)$ \\
\hline \multirow{2}{*}{$\begin{array}{l}\text { Lesão por dermatite } \\
\text { associada à incontinência }\end{array}$} & Sim & $14(58,3)$ \\
\hline & Não & $10(41,7)$ \\
\hline \multirow{2}{*}{ Lesão por fricção } & Sim & $4(16,7)$ \\
\hline & Não & $20(83,3)$ \\
\hline
\end{tabular}

Quanto às características das fitas cirúrgicas utilizadas pelos participantes, a fita microporosa foi utilizada por todos os pacientes e quatro apresentaram lesão por adesivo médico. Dos seis em uso de filme transparente, dois evoluíram com lesão por adesivo médico.

Todos os participantes do estudo apresentavam pelo menos um tipo de incontinência, sendo 44,8\% (30) mista, 32,8\% (22) urinária e 22,4\% (15) fecal, e 14 desenvolveram DAI mesmo com a utilização de algum dispositivo para seu manejo. A Tabela 3 apresenta a distribuição dos dispositivos para controle da incontinência e características da eliminação intestinal e urinária.

Os produtos utilizados pelos participantes na pele da região de fralda, para prevenção da dermatite, estão apresentados na Tabela 4. Percebeu-se que alguns pacientes utilizaram mais de um produto para prevenção de DAI.

A higienização da pele para troca de fralda era realizada com água e sabão neutro. Para proteção da pele, a maioria utilizava ácidos graxos essenciais (57/85\%).

Os pacientes que desenvolveram DAI estavam em uso de produto para proteção da pele: 14 (100\%) ácidos graxos essenciais e nove $(64,3 \%)$ óxido de zinco. Segundo

Tabela 3. Dispositivos para controle da incontinência e características da eliminação intestinal e urinária ( $n=67)$, Belo Horizonte, estado de Minas Gerais, 2018.

\begin{tabular}{|c|c|c|c|}
\hline \multirow{2}{*}{$\begin{array}{l}\text { Dispositivos para controle da } \\
\text { incontinência }\end{array}$} & \multirow{2}{*}{ Categoria } & \multicolumn{2}{|c|}{ Dermatite associada à incontinência (\%) } \\
\hline & & Não $(n=53)$ & $\operatorname{Sim}(n=14)$ \\
\hline \multirow{2}{*}{ Cateter urinário externo } & $\operatorname{Sim}$ & $5(9,4)$ & $1(7,1)$ \\
\hline & Não & $48(90,6)$ & $13(92,9)$ \\
\hline \multirow{2}{*}{ Cateter vesical de demora } & Sim & $33(62,3)$ & $5(35,7)$ \\
\hline & Não & $20(37,7)$ & $9(64,3)$ \\
\hline \multirow{2}{*}{ Fralda descartável } & Sim & $53(100,0)$ & $14(100,0)$ \\
\hline & Não & $0(0,0)$ & $0(0,0)$ \\
\hline Número de trocas de fralda & 4 & $42(79,3)$ & $12(85,7)$ \\
\hline descartável por dia & 6 & $11(20,7)$ & $2(14,3)$ \\
\hline \multirow{2}{*}{$\begin{array}{c}\text { Dispositivo coletor para estomia } \\
\text { intestinal }\end{array}$} & Sim & $3(5,6)$ & $1(7,1)$ \\
\hline & Não & $50(94,4)$ & $13(92,9)$ \\
\hline \multirow{4}{*}{$\begin{array}{c}\text { Dispositivo coletor para estomia } \\
\text { urinário }\end{array}$} & Sim & $2(3,7)$ & $2(14,3)$ \\
\hline & Não & $51(96,3)$ & $12(85,7)$ \\
\hline & 0 & $2(3,8)$ & $0(0,0)$ \\
\hline & 1 & $21(39,6)$ & $0(0,0)$ \\
\hline \multirow[t]{4}{*}{ Episódios de fezes ao dia } & 2 & $20(37,7)$ & $1(7,1)$ \\
\hline & 3 & $8(15,1)$ & $(21,4)$ \\
\hline & $>4$ & $2(3,8)$ & $10(71,5)$ \\
\hline & Pastosa & $33(62,3)$ & $0(0,0)$ \\
\hline \multirow[t]{2}{*}{ Consistência das fezes } & Semipastosa & $11(20,7)$ & $2(14,3)$ \\
\hline & Líquida & $9(17,0)$ & $12(85,7)$ \\
\hline
\end{tabular}


Tabela 4. Produtos utilizados na instituição para a pele para prevenção de dermatite associada à incontinência (DAI) ( $n=67$ ), Belo Horizonte, estado de Minas Gerais, 2018.

\begin{tabular}{|c|c|c|c|}
\hline \multirow[t]{2}{*}{ Variável } & \multirow[t]{2}{*}{ Categoria } & \multicolumn{2}{|c|}{$\begin{array}{c}\text { Dermatite associada à } \\
\text { incontinência (\%) }\end{array}$} \\
\hline & & Não (n = 53) & $\operatorname{Sim}(n=14)$ \\
\hline \multirow{2}{*}{ Óxido de zinco } & Sim & $28(52,8)$ & $9(64,3)$ \\
\hline & Não & $25(47,2)$ & $5(35,7)$ \\
\hline Ácidos graxos & Sim & $43(81,1)$ & $14(100,0)$ \\
\hline essenciais & Não & $10(18,9)$ & $0(0,0)$ \\
\hline \multirow{2}{*}{ Nistatina } & $\mathrm{Sim}$ & $5(9,4)$ & $3(21,4)$ \\
\hline & Não & $48(90,6)$ & $11(78,6)$ \\
\hline \multirow{2}{*}{ Cetoconazol } & Sim & $3(5,6)$ & $1(7,2)$ \\
\hline & Não & $50(94,4)$ & $13(92,8)$ \\
\hline \multirow{2}{*}{ Dexpantenol } & $\mathrm{Sim}$ & $20(37,7)$ & $1(7,2)$ \\
\hline & Não & $33(62,3)$ & $13(92,8)$ \\
\hline
\end{tabular}

os profissionais, é comum a realização da mistura dos dois produtos para aplicação em região de fralda.

Considerando os 24 participantes que apresentaram LC, um manteve pele íntegra ao redor da lesão. Os 23 (87,5\%) restantes apresentavam algum tipo de alteração, como, por exemplo, maceração (8/33,3\%), descamação (6/25\%), eritema $(5 / 20,8 \%)$ e desidratação (4/16,7\%). O edema local estava presente em $15(62,5 \%)$ participantes, com classificação de $1+(6 / 40 \%), 2+(5 / 33,3 \%)$ e $3+(4 / 26,7 \%)$.

\section{DISCUSSÃO}

Destaca-se que nas bases de dados há ausência de publicações a respeito da incidência e prevalência das LCs DAI, LF e lesão relacionada a adesivo médico - consideradas em conjunto. Neste estudo, a incidência estimada dessas lesões foi de 35,8\%, com média de 11,0 ( \pm 13,9) dias e mediana de 5,0 dias para seu surgimento. Esses dados são surpreendentes, portanto, essas lesões não podem continuar a ser ignoradas pelos profissionais da prática clínica e pelos gestores das instituições hospitalares.

Estudo de coorte prospectivo realizado por Konya et al. ${ }^{8}$ com 155 indivíduos idosos com idade igual ou superior a 65 anos em uma instituição de longa permanência encontrou incidência de lesão por adesivo médico de 15,5\%. Já um estudo de coorte prospectivo em uma clínica vascular durante um período de três meses, com acompanhamento de 120 pacientes, encontrou incidência de lesão por adesivo médico de 5,8\% ${ }^{9}$, dados semelhantes em comparação ao deste estudo. Apesar das diferenças entre a UTI onde o estudo foi realizado, a instituição de longa permanência e a clínica vascular, os estudos realizados nos três cenários contaram com predomínio de participantes idosos, com mais de 60 anos, e com grau de dependência, dificultando o autocuidado, aumentando, assim, a chance de ocorrência das LCs.

Em estudo de revisão sistemática sobre as lesões por fricção, publicado em 2015, não foram encontrados estudos primários em pacientes $\operatorname{críticos}^{10}$, apenas estudos de prevalência de lesões por fricção realizados em instituições de longa permanência. Estudo desenvolvido em instituições de longa permanência no Canadá, com 113 idosos, identificou prevalência de LF de 22\% ${ }^{11}$.Já em estudo realizado no Japão, a prevalência variou de 3 a $9 \%$ em um total de 410 pessoas com idade entre 73 e $87 \operatorname{anos}^{12}$. No Brasil, um estudo com pacientes oncológicos obteve prevalência de LF de 3,3\% e a maioria era idosos do sexo feminino ${ }^{13}$.

Adesivos médicos e curativos adesivos foram identificados como os principais responsáveis pelas lesões por adesivo médico nos extremos de idade. Apesar de idosos e neonatos constituírem os maiores grupos de risco, existem outros grupos sujeitos à fragilização, como indivíduos em estado crítico, em cuidados paliativos e em tratamento antineoplásico ${ }^{10}$.

Todos os pacientes da amostra tinham câncer e haviam sido submetidos a tratamento neoadjuvante, seja com quimioterapia, radioterapia ou quimioterapia associada à radioterapia. Sabe-se que o uso de agentes antineoplásicos acarreta alterações na epiderme, na derme e na síntese de colágeno. Reações cutâneas são frequentes nesses indivíduos, além do retardo no processo de cicatrização ${ }^{1}$.

Dado importante identificado nos participantes foi o predomínio de desnutrição. Por se tratar de pacientes oncológicos, esse dado não ocasionou surpresa, visto que o câncer altera as funções fisiológicas do organismo e causa desequilíbrio no metabolismo, no qual a quebra de proteínas e lipídios fica acelerada, enquanto a síntese de proteínas musculares fica diminuída. Essas alterações contribuem para o aumento do gasto energético e podem resultar em perda ponderal progressiva ${ }^{14}$. A maioria apresentou alterações nas taxas de hemoglobina sérica e glicemia capilar.

A hemoglobina sérica e o IMC são marcadores nutricionais simples e devem ser verificados continuamente nos pacientes em risco nutricional a fim de reduzir complicações futuras. A cicatrização é um processo dinâmico, com influência direta do estado nutricional. $\mathrm{O}$ reparo e a remodelação tissular necessitam de suplemento 
suficiente de precursores, cofatores e fonte de energia. A desnutrição afeta negativamente a evolução clínica dos indivíduos por aumentar o tempo internação, a incidência de complicações pós-operatórias, como infecções e retardo na epitelização de feridas, além de aumentar a taxa de mortalidade ${ }^{15}$. Condições clínicas tais como caquexia/ anorexia, desnutrição, anemias, síndromes metabólicas, idade avançada e distúrbios circulatórios comprometem a saúde da pele ${ }^{16}$.

O principal motivo de internação dos pacientes na UTI foi insuficiência respiratória aguda e as doenças associadas prevalentes entre os pacientes com LC foram hipertensão arterial sistêmica, cardiopatia e diabetes melito. Essa última é uma das principais causas de interferência nos processos de cicatrização de feridas, pois o paciente apresenta comprometimento do sistema imunológico e maior propensão à ocorrência de infecções ${ }^{15}$.

Alguns hábitos nocivos à saúde, como etilismo, tabagismo, alimentação inadequada e sedentarismo, estão intimamente ligados à ocorrência das doenças crônicas não transmissíveis e das lesões cutâneas ${ }^{13}$. Outros fatores que acarretam alteração no processo de cicatrização são as doenças crônicas, a idade e o tratamento com agentes antineoplásicos ${ }^{13}$. No estudo realizado, a minoria dos pacientes era etilista e tabagista.

A ocorrência das seis lesões por adesivo médico deste estudo culminou com a mudança na marca da fita microporosa utilizada na instituição, além da padronização de uma fita para pele sensível, já que a maioria dos pacientes era idoso e submetido a tratamento neoadjuvante.

Dentre as principais causas para o surgimento de lesões por fricção, destacam-se os pacientes debilitados e totalmente dependentes para as atividades de vida diária, com nutrição e mobilidade prejudicadas, pele friável, em uso de corticoides, anti-inflamatórios e antihipertensivos, idosos e em cuidados paliativos ${ }^{6,11,12,17}$.

A DAI é um processo inflamatório causado pelo dano da matriz água-lipídio-proteína da pele resultante da exposição prolongada ao agente irritativo ${ }^{16}$, sua ocorrência está relacionada à idade, câncer, número de trocas de fraldas por dia, eritema, uso de hipnóticos/ sedativos, antipsicóticos, doenças crônicas, incontinência dupla, incontinência fecal, oxigenação prejudicada, febre, desnutrição, diminuição da perfusão e oxigenação, alterações cutâneas e idade ${ }^{1}$. A maioria desses fatores estava presente nos participantes do estudo realizado na UTI e a incidência de DAI foi de 20,8\%.

Dados diferentes foram encontrados em estudo nacional realizado com pacientes críticos em um hospital privado no Paraná. A incidência de DAI foi de 15,27\%, com maioria dos pacientes com idade acima de 60 anos e do sexo feminino ${ }^{18}$. Entretanto, em estudo realizado em uma UTI geral com 145 pacientes adultos, o autor identificou incidência de $26,2 \%$ de DAI e tempo médio de 14 dias para seu surgimento. A maioria dos pacientes era do sexo masculino e com mediana de idade de $69 \operatorname{anos}^{19}$.

Os resultados deste estudo corroboram outro cujos resultados confirmaram que $87 \%$ dos pacientes brancos do sexo feminino desenvolveram DAI ${ }^{16}$. A pele negra parece fator protetor de lesões cutâneas, pela sua espessura, o que a torna mais resistente ${ }^{10}$.

No cenário de instituição de longa permanência, a incidência de DAI foi de $8 \%$ inferior a obtida neste estudo $^{20}$.

A maioria dos pacientes do estudo estava em uso de dispositivos para incontinência urinária, como cateter vesical de demora e fralda descartável. Esses dados também estavam presentes no estudo brasileiro realizado com pacientes adultos com objetivo de avaliar as principais tecnologias aplicadas pela enfermagem no controle urinário, que identificou que $60 \%$ dos pacientes usavam fraldas, seguido de drenagem por cateterismo intermitente $(24,8 \%)^{21}$.

A pele da região perineal de todos os pacientes era higienizada durante as trocas de fraldas com água mais sabão neutro. $O$ uso contínuo de sabões neutros e demais substâncias para a higiene causa alteração na estrutura de barreira da pele, o que leva ao ressecamento, além aumentar o $\mathrm{pH}$ da pele, que é levemente ácido ${ }^{5}$.

A fricção da pele para remoção de resíduos irritantes, como pomadas, cremes gordurosos e óleos, também provoca perturbações na função barreira da pele por meio da ação mecânica ${ }^{5}$. Ou seja, além da agressão da pele dos pacientes da amostra com o contato da urina e fezes, havia também a agressão pelo produto utilizado para higiene e fricção para remoção dos ácidos graxos essenciais e da pomada à base de óxido de zinco.

No estudo, identificou-se que a maioria dos participantes utilizava fralda descartável, que era trocada quatro vezes ao dia, mesmo nos pacientes com mais 
episódios de fezes líquidas por dia. Essa ação tem relação com o contrato com a saúde suplementar, entretanto, quando a região de fraldas é mantida exposta ao contato com as eliminações urinária e intestinal, aumenta o risco de o paciente desenvolver DAI.

O consenso internacional sobre DAI classifica como protetores cutâneos apenas a pomada à base de vaselina (não disponível no Brasil), a dimeticona e o acrilato líquido ${ }^{4}$. Entretanto, muitos participantes do estudo utilizavam os ácidos graxos essenciais para prevenção da dermatite, apesar de esse produto ser classificado como emoliente, devendo ser utilizado apenas para evitar a perda da umidade pela pele ${ }^{5}$.

Além do mais, os ácidos graxos essenciais são absorvidos pela fralda descartável, assim como qualquer substância líquida, reduzindo a capacidade de absorver efluentes e mantendo a pele exposta à umidade por um período maior. No presente estudo, os ácidos graxos essenciais foram utilizados indiscriminadamente, até mesmo misturados a outros produtos, o que pode ter colaborado para a manutenção da pele exposta à umidade por um período maior.

O estudo teve limitações relacionadas, principalmente, à sua casuística, além de ser realizado em apenas um hospital especializado em oncologia, com tempo de coleta de apenas um mês, o que dificultou, também, a realização de análises estatísticas mais robustas. Por se tratar de um estudo piloto, pretende-se, na próxima etapa, coletar dados durante 12 meses, mantendo seguimento de 30 dias. Recomenda-se replicar essa pesquisa em outros hospitais oncológicos, uma vez que estudos de incidência permitirão avaliar os fatores preditores para ocorrência de LC nessa clientela.
O conhecimento produzido com a realização deste estudo poderá contribuir para o fortalecimento da prática clínica de enfermeiros na avaliação de seus pacientes e na diferenciação das LCs das LPs.

\section{CONCLUSÃO}

A incidência das LCs foi elevada $(35,6 \%)$ nos pacientes oncológicos internados em UTI, infere-se que tenha relação com a presença do câncer e o tratamento neoadjuvante, o que requer estudos específicos para elucidação. Foram consideradas LCs a DAI, a lesão por adesivo médico e a LF, com respectiva incidência de 20; $8 ; 8 ; 9$ e 5,9\%. Todos os participantes utilizavam algum tipo de adesivo e dispositivo para incontinência. As lesões surgiram em média 11 dias após a internação na UTI. Também foi possível descrever o perfil demográfico e clínico dos pacientes da amostra e caracterizar as lesões e a pele ao redor.

\section{CONTRIBUIÇÃO DOS AUTORES}

Conceitualização, Pires Júnior JF e Borges EL; Metodologia, Pires Júnior JF e Borges EL; Investigação, Pires Júnior JF e Borges EL; Redação - Primeira versão, Pires Júnior JF; Borges EL e Spira JAO; Redação Revisão \& Edição, Pires Júnior JF; Borges EL e Spira JAO; Supervisão, Borges EL.

\section{REFERÊNCIAS}

1. Gray M, Black JM, Baharestani MM, Bliss DZ, Colwell JC, Goldberg M, et al. Moisture-associated skin damage. J Wound Ostomy Continence Nurs. 2011;38(3),233-41. https://doi.org/10.1097/won.0b013e318215f798.

2. National Pressure Ulcer Advisory Panel. Pressure ulcer stages revised [Internet]. Washington, DC: NPUAD; 2016. [citado 14 Fev 2019]. Disponível em: http://www.npuap. org/resources/educational-and-clinical-resources/npuappressure-injury-stages/.

3. Caliri MHL, Santos VLCG, Mandelbaum MHS, Costa IG. Classificação das lesões por pressão - Consenso NPUAP 2016 - Adaptada culturalmente para o Brasil [Internet]. São Paulo: Associação Brasileira de Estomaterapia/Associação Brasileira de Enfermagem em Dermatologia; 2016 [citado 14 Fev 2019]. Disponível em: http://www.sobest.org.br/ textod/35
4. Ministério da Saúde (BR). Portaria No 529 de 01 de abril de 2013 [Internet]. Brasília, DF; 2013. [citado 16 Jul 2016]. Disponível em: http://bvsms.saude.gov.br/bvs/saudelegis/g m/2013/prt0529 0104 2013.html

5. Beeckman D. A decade of research on incontinenceassociated dermatitis (IAD): evidence, knowledge gaps and next steps. J Tissue Viability. 2016;26(1):47-56. https://doi. org/10.1016/j.jtv.2016.02.004

6. Strazzieri-Pulido KC, Peres GR, Campanili TC, Santos VLG. Incidence of skin tears and risk factors: a systematic literature review. J Wound Ostomy Continence Nurs. 2017;44(1):2933. https://doi.org/10.1097/WON.0000000000000304

7. McNichol L, Lund C, Rosen T, Gray M. Medical adhesives and patient safety: state of the science: consensus statements for the assessment, prevention, and treatment of adhesive-related skin injuries. I Wound Ostomy 
Continence Nurs. 2013;32(5):365-80. https://doi.org/10.1097/ NOR.0b013e3182a39caf.

8. Konya C, Sanada $H$, Sugama J, Okuwa M, Kamatani $Y$, Nakagami G, et al. Skin injuries caused by medical adhesive tape in older people and associated factors. J Clin Nurs. 2010;9(10):1236-42. https://doi.org/10.1111/j.1365-2702. 2009.03168.x

9. Ratliff, CR. Descriptive study of the frequency of medical adhesive-related skin injuries in a vascular clinic. J Vasc Nurs. 2017;35(2):86-9. https://doi.org/10.1016/j.jvn.2017.01.001

10. Strazzieri-Pulido KC, Peres GRP, Campanili TCGF, Santos VLCG. Skin tear prevalence and associated factors: a systematic review. Rev Esc Enferm USP. 2015;49(4):674-80. https://doi.org/10.1590/S0080-623420150000400019

11. LeBlanc K, Christensen D, Cook J, Culhane B, Gutierrez O. Prevalence of skin tears in a long-term care facility. J Wound Ostomy Continence Nurs. 2013;3(40):580-4. https://doi. org/10.1097/WON.0b013e3182a9c111

12. Koyano Y, Nakagami G, lizaka S, Minematsu T, Noguchi H, Tamai N, et al. Exploring the prevalence of skin tears and skin properties related to skin tears in elderly patients at a longterm medical facility in Japan. Int Wound J. 2014;13(2):18997. https://doi.org/10.1111/iwj.12251

13. Amaral AFS, Pulido KCS, Santos VLCG. Prevalence of skin tears among hospitalized patients with cancer. Rev Esc Enferm USP. 2012;46(Espc):44-50. https://doi.org/10.1590/ S0080-62342012000700007

14. Machry VR, Susin CF, Barros RC, Lago LD. Desnutrição em pacientes com câncer avançado: uma revisão com abordagem para o clínico. Rev AMRIGS. 2011;55(3):296-301. https://doi.org/10.1590/50080-62342012000700007
15. Ramneesh G, Sheerin S, Surinder S, Bir S. A prospective study of predictors for post laparotomy abdominal wound dehiscence. J Clin Diagn Res. 2014;8(1):80-3. https://doi. org/10.7860/JCDR/2014/7348.3921

16. Bliss DZ, Savik K, Thorson MA, Ehman SJ, Lebak K, Beilman G. Incontinence-associated dermatitis in critically ill adults. J Wound Ostomy Continence Nurs. 2011;38(4),433-45. https://doi.org/10.1097/WON.0b013e318220b703

17. Holmes RF, Davidson MW, Bonnie J. Skin tears care and management of the older adult at home. Home Healthcare Nurse. 2013;31(2):90-101. https://doi.org/10.1097/ NHH.0b013e31827f458a

18. Baessa CEB, Meireles VC, Balan MAJ. Ocorrência de dermatite associada à incontinência em pacientes internados na unidade de terapia intensiva. ESTIMA, Braz J Enterostomal Ther. 2014;12(2) [citado 30 Nov 2017]; Disponível em: https://www.revistaestima.com.br/index.php/estima/article/ view/89

19. Valls-Matarín J, Del Cotillo-Fuente M, Ribal-Prior R, PujolVila M, Sandalinas-Mulero I. Incidence of moistureassociated skin damage in an intensive care unit. Enferm Intensiva. 2017;28(1):13-20. https://doi.org/10.1016/j. enfi.2016.11.001

20. Campbell JL, Coyer FM, Osborne, SR. Incontinenceassociated dermatitis: a cross-sectional prevalence study in the Australian acute care hospital setting. Int Wound J. 2014;13(3):403-11. https://doi.org/10.1111/iwj.12322

21. Bitencourt GR, Alves LAF, Santana RF. Practice of use of diapers in hospitalized adults and elderly: cross-sectional study. Rev Bras Enferm. 2018;71(2):343-9. https://doi. org/10.1590/0034.7167-2016-0341 